\title{
Long-term outcomes of metachronous neoplasms in the ileal pouch and rectum after surgical treatment in patients with familial adenomatous polyposis
}

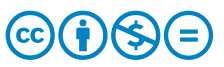

\begin{abstract}
Authors
Masahiro Tajika', Tsutomu Tanaka', Makoto Ishihara ${ }^{1}$, Yutaka Hirayama', Sachiyo Oonishi ${ }^{1}$, Nobumasa Mizuno ${ }^{2}$, Takamichi Kuwahara², Nozomi Okuno ${ }^{2}$, Shinpei Matsumoto ${ }^{2}$, Taihei Ooshiro ${ }^{3}$, Takashi Kinoshita ${ }^{3}$, Koji Komori ${ }^{3}$, Vikram Bhatia ${ }^{4}$, Kazuo Hara², Yasushi Yatabe ${ }^{5}$, Yasumasa Niwa' ${ }^{1}$
\end{abstract}

Institutions

1 Department of Endoscopy, Aichi Cancer Center Hospital, Nagoya, Japan

2 Department of Gastroenterology, Aichi Cancer Center Hospital, Nagoya, Japan

3 Department of Gastroenterological Surgery, Aichi Cancer Center Hospital, Nagoya, Japan

4 Department of Medical Hepatology, Institute of Liver and Biliary Sciences, New Delhi, India

5 Department of Pathology and Molecular Diagnostics, Aichi Cancer Center Hospital, Nagoya, Japan

submitted 28.9.2018

accepted after revision 27.12.2018

Bibliography

DOI https://doi.org/10.1055/a-0849-9465 |

Endoscopy International Open 2019; 07: E691-E698

(c) Georg Thieme Verlag KG Stuttgart · New York

eISSN 2196-9736

Corresponding author

Masahiro Tajika, MD, PhD, Department of Endoscopy, Aichi

Cancer Center Hospital, 1-1 Kanokoden, Chikusa-ku,

Nagoya 464-8681, Japan

Fax: $+81-52-763-5233$

mtajika@aichi-cc.jp

\section{ABSTRACT}

Background and study aims Restorative proctocolectomy has become the most common surgical option for patients with familial adenomatous polyposis (FAP). However, adenomas and even carcinomas may develop in the ileal pouch over time. The aim of this study was to evaluate the long-term incidence and nature of ileal pouch or distal ileal adenomas and carcinomas in patients with FAP.

Patients and methods This was a retrospective study of 47 FAP patients with Kock's continent ileostomy (Kock) (n $=8)$, ileorectal anastomosis (IRA) $(n=13)$, and ileal pouchanal anastomosis (IPAA) $(n=26)$. Patients were followed with a standardized protocol including chromoendoscopy and biopsies of visible polyps in the ileal pouch, distal ileum, and rectum every 6 to 12 months.

Results Median follow-up was 21.0 years. Overall risk of adenoma development was significantly higher in IRA patients, with incidence rates of $85 \%$ and $100 \%$ at 5 and 10 years' follow-up, respectively, compared to pouch patients $($ Kock + IPAA) $(P<0.001)$. However, there was also a high frequency of adenomas in the ileal pouch mucosa, with rates of $12 \%, 33 \%$, and $68 \%$, at 5,10 , and 20 years of follow-up, respectively. Maximum size of ileal pouch adenomas was significantly related to time since surgery $(P=$ $0.0214)$. Six cases of advanced adenomas including two cases of adenocarcinomas developed in the ileal pouch mucosa.

Conclusions There is a significant incidence of adenoma (s) in the ileal pouch of FAP patients on long-term followup. Regular endoscopic surveillance is recommended, not only in IRA patients, but also in pouch patients after proctocolectomy.

\section{Introduction}

Familial adenomatous polyposis (FAP) is an inherited, autosomal-dominant disease caused by a germline mutation of the adenomatous polyposis coli gene (APC) [1]. The phenotype is characterized by development of hundreds of colorectal adenomas, leading to a $100 \%$ life-time risk of colorectal cancer [2]. For this reason, a restorative proctocolectomy with ileal pouch-anal anastomosis (IPAA) [3-6] is recommended for patients with FAP to prevent development of colorectal cancer. 
However, because patients with FAP are generally young and often asymptomatic at the time of surgery, proctocolectomy is not easy to accept from functional and social perspectives.

Another widely accepted surgical procedure is colectomy with ileorectal anastomosis (IRA), performed in patients with a low risk of rectal cancer, especially in female patients who wish to have children. The major advantage of IRA is preservation of rectal innervation and subsequent better quality of life. However, continuing endoscopic surveillance for adenomas in the rectum is necessary, and a $13 \%$ to $25 \%$ cumulative risk of rectal cancer is reported after 15 to 25 years despite surveillance [79]. On the other hand, IPAA was theorized to eliminate risk of colorectal cancer and adenomas and, perhaps, need for further lower gastrointestinal surveillance. However, in 1982, Beart et al. [10] first described a FAP patient with a Kock pouch continent ileostomy, in whom a large sessile tubulovillous adenoma and multiple smaller adenomatous polyps developed. Since then, there have been around 30 reports of ileal pouch adenomas developing in these patients [4,9-22], and the necessity for endoscopic surveillance for patients with IPAA is becoming recognized. Furthermore, there are several reports of cancers arising from the ileal pouch mucosa, as opposed to the anastomotic site, in patients with FAP $[15-17,19,20]$. It is clear that adenomas or carcinomas develop not only in the residual rectal mucosa after IRA, but also in the ileal pouch mucosa after IPAA. However, long-term outcomes of metachronous adenomas and carcinomas in the ileal pouch after surgical treatment in patients with FAP are still unclear. In addition, there is little information about when endoscopic surveillance for the ileal pouch should start, and how much of the ileal mucosa should be examined, only the ileal pouch or also the distal ileum?

The aim of this study was to determine the prevalence and nature of adenomas and carcinomas developing in ileal pouch mucosa, rectum, and distal ileal mucosa of the afferent limb in patients with FAP after surgery.

\section{Patients and methods}

Endoscopic and medical records of all patients with FAP treated in Aichi Cancer Center Hospital (ACCH), Nagoya, Japan, between January 1965 and December 2017 were reviewed. FAP was defined by presence of more than 100 colorectal adenomas (all patients) and/or a family history of FAP. Patients who had at least one endoscopic examination during follow-up were eligible for this study. In general, it was recommended that IRA patients have endoscopic examinations every 6 months, and that Kock's pouch and IPAA patients have annual examinations after surgery. The IPAA patients underwent anal mucosectomy leaving a short rectal muscular cuff above the dentate line and a trans-anal hand-sewn ileoanal anastomosis. Pouch construction with two ileal limbs, $15 \mathrm{~cm}$ in length (J-pouch), was used. In this study, patients who had undergone pouch construction, either Kock or IPAA, were defined as pouch patients. Patient demographic data, surgical data, details of pathological specimens, and details of upper gastrointestinal endoscopy were obtained from the medical records. The study was approved by the ethics committee of $\mathrm{ACCH}$, and all patients provided their informed consent for collection and subsequent use of data for research purposes. The study was carried out in accordance with the Helsinki Declaration.

The interval between surgery and adenoma appearance was defined as the time from surgery to the first report showing histologically confirmed adenomas in the ileal mucosa. Number, size, and histology of adenomas occurring in the ileal mucosa were determined based on the first report in which an adenoma had been histologically confirmed or the last report before treatment. For each patient, the most advanced histologic diagnosis was taken as valid. The endoscopic examination protocol was as follows. All patients were administered $1 \mathrm{~L}$ of polyethylene glycol-electrolyte solution on the morning of the examination. Scopolamine butylbromide $(10 \mathrm{mg})$ or glucagon $(0.5 \mathrm{mg})$ was administered intravenously in patients without contraindications to reduce bowel movements. A flexible endoscope (GIF H260, GIF H260Z, GIF H290Z; Olympus Optical Co. Ltd., Tokyo, Japan) was used for the examination. In addition to a thorough examination of the pouch in Kock or IPAA patients and rectum in IRA patients, the distal 25 to $30 \mathrm{~cm}$ of the afferent limb and the anal canal were also examined carefully. In this study, the distal ileal mucosa of the afferent limb was defined as pre-pouch ileal mucosa. After initial complete examination with white light imaging, chromoendoscopy using $0.2 \%$ indigo carmine solution was performed, using a spray catheter (PW-5L-1; Olympus Optical Co. Ltd.). If a polyp was detected, magnifying endoscopy was performed to evaluate Kudo's pit pattern classification. Polyps were classified into three size groups by diameter: 1 to $4 \mathrm{~mm}, 5$ to $9 \mathrm{~mm}$, and $\geq 10 \mathrm{~mm}$. Advanced adenomas were defined as adenomas $\geq 10 \mathrm{~mm}$ in greatest diameter and/or with high-grade dysplasia. Polyp size was estimated using the diameter of the endoscope or the width of biopsy forceps.

\section{Statistical analysis}

Differences in proportions between patients were compared by Pearson's chi-squared test. The Mann-Whitney $U$ test was used to compare differences between medians. The Kaplan-Meier method was used to calculate cumulative incidence rates, and differences were analysed by the log-rank test. Spearman's rank correlation coefficient and single linear regression analysis were used to study the relationship between maximum ileal pouch adenoma size and time since pouch surgery. A $P$ value < 0.05 was considered significant. Data files were analysed using JMP 12 (SAS Institute Inc., Cary, North Carolina, United States).

\section{Results}

Eighty-four patients with FAP were treated in our hospital between January 1965 and December 2016; of them, 47 patients from 36 families (27 females; median age: 52.0 years; age range: $24-80$ years) were eligible for this study. Fourteen patients had undergone Kock and IRA up to May 1987. After March 1988, 32 patients had undergone IPAA, and one patient with advanced cancer in the lower rectum had undergone Kock. - Table 1 lists characteristics of the pouch patients (Kock and IPAA) and IRA patients, as well as details of endoscopic surveil- 
- Table 1 Characteristics of pouch patients and IRA patients and details of endoscopic surveillance.

\begin{tabular}{|c|c|c|c|}
\hline & Pouch patients & IRA patients & \\
\hline Factor & $(n=34)$ & $(n=14)$ & $P$ value \\
\hline Median age at surgery, y (range) & $34.6(17-52)$ & $36.7(19-67)$ & 0.2630 \\
\hline Sex female, n (\%) & $18(52.9)$ & $10(71.4)$ & 0.2480 \\
\hline \multicolumn{4}{|l|}{ Median polyp count at surgery } \\
\hline - Total & $648(105-20000)$ & $570(100-9436)$ & 0.1803 \\
\hline - Colon & $1970(77-17200)$ & $420(80-9340)$ & 0.2523 \\
\hline - Rectum ${ }^{1}$ & $200(5-5282)$ & $70(1-1071)$ & 0.1686 \\
\hline Gastric polyp, n (\%) & $26(76.5 \%)$ & $11(84.6 \%)$ & 0.5417 \\
\hline Papillary adenoma, n (\%) & $16(47.0 \%)$ & $5(38.5 \%)$ & 0.5959 \\
\hline Extrapapillary adenoma, n (\%) & $15(44.1 \%)$ & $6(46.2 \%)$ & 0.9000 \\
\hline Median follow-up period, y (range) & $21.6(3.7-38.8)$ & $17.3(1-37.8)$ & 0.7662 \\
\hline \multicolumn{4}{|l|}{ Median duration to 1st endoscopy } \\
\hline - after surgery, months (range) & $108.6(12-305)$ & $8.4(5-17)$ & $<0.001$ \\
\hline \multicolumn{4}{|l|}{ Median duration of endoscopic surveillance } \\
\hline - after 1st endoscopy, months (range) & $12(6-60)$ & $6(6-12)$ & 0.0385 \\
\hline \multicolumn{4}{|l|}{ Median time of endoscopic surveillance } \\
\hline - after 1st endoscopy, n (range) & $9(3-38)$ & $11(1-56)$ & 0.2456 \\
\hline
\end{tabular}

lance. There were no significant differences in age at the time of surgery, sex, median polyp count at surgery, and co-existence of gastric polyps, papillary adenomas, or extra-papillary adenomas between pouch patients and IRA patients.

Median follow-up of all patients was 21.0 years (range: 1 38.8 years). Although median duration to first endoscopy after surgery and median duration of endoscopic surveillance after first endoscopy were significantly longer in the pouch patients than in the IRA patients ( $P<0.001$ and $P=0.0385$, respectively), there was no significant difference in median time of endoscopic surveillance after first endoscopy.

Maximum size, number, shape, and histology of polyps found in each patient and the age of the pouch are shown in - Table 2. In pouch patients, adenomas developed in 24 of 34 patients $(70.6 \%)$, ranging in number from one to more than 300 . Adenoma size ranged from 2 to $40 \mathrm{~mm}$. Two cases of adenocarcinoma and six cases of advanced adenoma developed in the pouch patients. These tumors developed in the ileal pouch mucosa itself, as opposed to the ileoanal anastomosis site. In IRA patients, from one to 20 adenomas were observed in all cases in the rectum, and their sizes varied from 2 to $10 \mathrm{~mm}$.

Risk of rectal adenoma development after colectomy with IRA was $85 \%$ and $100 \%$ at 5- and 10-year follow-up, respectively. This was significantly higher than risk of adenomas in ileal pouch patients $(P<0.001)$. However, risk of adenoma development in the ileal pouch was also substantial, with $12 \%, 33 \%$, $52 \%$, and $68 \%$ of pouch patients (Kock's pouch and IPAA) devel- oping adenomas at 5, 10, 15, and 20 years of follow-up, respectively ( $\triangleright$ Fig. 1 ).

Tiny adenomas of 1 to $3 \mathrm{~mm}$ were observed in the pre-pouch ileal mucosa in four of 24 patients (16.7\%). However, no patient had adenomas in the ileal mucosa above the IRA site. Prevalence of ileal adenoma was significantly higher in the ileal pouch mucosa than in the pre-pouch ileal mucosa $(P<0.001)$. Risk of adenoma development in the pre-pouch after surgery was $4.4 \%$ and $36 \%$ at 20 - and 30 -year follow-up, respectively, and it was significantly lower than risk of ileal pouch adenomas $(P<$ 0.001) ( Fig. 2).

There was a positive correlation between maximum size of ileal pouch adenomas and time since surgery in pouch patients $(r=0.4671, P=0.0214)$. Linear regression analysis ( $\mathbf{F i g . 3}$ ) showed the positive relationship between maximum size of ileal pouch adenomas and time since surgery as: Adenoma size $(\mathrm{mm})=0.29+0.69 \times$ time $(y)$.

\section{Discussion}

IPAAs have been used for patients with FAP after proctocolectomy because they theoretically eliminate risk of colorectal cancer and adenomas and need for further lower gastrointestinal surveillance. However, despite a reduction in colorectal cancer and adenomas after the surgery, occurrence of adenomas in the constructed pouch brings new concerns about surveillance and management $[23,24]$. In the current study, overall inci- 
- Table 2 Characteristics of polyps in pouch patients and IRA patients.

\begin{tabular}{|c|c|c|c|c|}
\hline & \multicolumn{2}{|c|}{ Pouch patients $(n=34)$} & \multicolumn{2}{|l|}{ IRA patients $(n=14)$} \\
\hline & $\begin{array}{l}\text { Ileal pouch mucosa } \\
(n=24)\end{array}$ & $\begin{array}{l}\text { Pre-pouch mucosa } \\
(n=4)\end{array}$ & Rectal mucosa $(n=14)$ & Ileal mucosa $(n=0)$ \\
\hline \multicolumn{5}{|l|}{ Maximum size of polyp, $n$} \\
\hline . $1-4 \mathrm{~mm}$ & 12 & 4 & 11 & 0 \\
\hline . $5-9 \mathrm{~mm}$ & 6 & 0 & 2 & 0 \\
\hline - $\geq 10 \mathrm{~mm}$ & 6 & 0 & 1 & 0 \\
\hline \multicolumn{5}{|l|}{ Number of polyps } \\
\hline . $<50$ & 10 & 4 & 14 & 0 \\
\hline . $\geq 50$ & 5 & 0 & 0 & 0 \\
\hline \multicolumn{5}{|l|}{ Shape of polyps } \\
\hline - Sessile & 23 & 4 & 14 & 0 \\
\hline - Semi-pedunculated & 1 & 0 & 0 & 0 \\
\hline \multicolumn{5}{|l|}{ Histology } \\
\hline - Low-grade dysplasia & 21 & 1 & 14 & 0 \\
\hline - High-grade dysplasia & 1 & 0 & 0 & 0 \\
\hline - Carcinoma & 2 & 0 & 0 & 0 \\
\hline Advanced adenoma & 6 & 0 & 0 & 0 \\
\hline Time since operation, y & $11.8 \pm 6.1$ & $23.1 \pm 5.8$ & $2.1 \pm 2.3$ & \\
\hline $\begin{array}{l}\text { Values are means } \pm \text { SD } \\
\text { IRA, ileorectal anastomosis. }\end{array}$ & & & & \\
\hline
\end{tabular}

dence of ileal pouch adenomas was as high as $70.6 \%$ in pouch patients at median follow-up of 21.0 years (range: $1-38.8$ years) after surgery. Cumulative risk of adenoma in the pouch was $12 \%, 33 \%$, and $68 \%$ at 5-, 10-, and 20 -year follow-up, respectively ( $\mathbf{F i g . 1}$ ). Incidence of pouch adenomas increases steadily with pouch age. These findings are similar to previous studies. There are approximately 30 reports of development of pouch adenomas [4,9-22], including three studies with more than 100 FAP patients $[15,16,18]$. Overall incidence of ileal pouch adenomas was $15.8 \%$ to $48.3 \%$ at median follow-up of 6.8 to 15 years after surgery. Furthermore, risk of adenoma development in the ileal pouch increased with time: $7 \%, 35 \%$, and $75 \%$ at 5-, 10-, and 15-year follow-up, respectively [4]. To the best of our knowledge, this is the first report of long-term follow-up of more than 20 years after surgery in patients with FAP. Several predictors that may favor development of pouch adenomas have been investigated, such as severity of duodenal polyposis $[11,13,17]$, presence of advanced duodenal adenoma prior to surgery [18], high polyp counts (>1000) at colectomy [17], and types and locations of APC mutations [13, 14, 25]. However, because incidence of pouch adenomas increased steadily according to time after surgery, most, if not all, of these patients are destined to develop adenomas. Hence, investigating predictors for pouch adenoma development may be meaningless. The problem is not whether patients with
IPAA develop pouch adenomas, but whether these adenomas harbor clinically relevant malignant potential.

In the current study, there were two cases of adenocarcinomas and six cases of advanced adenomas in pouch patients. To date, 22 cases of ileal pouch adenocarcinomas have been reported in the literature ( Table 3 ) $[15-17,19,20]$. Although several other cases of adenocarcinoma after restorative proctocolectomy seem to have arisen from residual rectal mucosa at the ileoanal anastomosis [26], these pouch cancers have clearly appeared in the ileal pouch, and not in the anal transit zone. Until now, malignant potential of pouch adenomas and lifetime risk of pouch cancer for patients with FAP have not been clear. Friederich et al. [15] reported a cumulative risk of pouch adenocarcinoma of $1 \%$ after 10 years in their cohort of 212 FAP patients. If ileal pouch adenomas progress to carcinoma following a similar pattern seen in the colon, factors that may determine risk of malignant transformation are number of polyps, large size, severity of dysplasia, and villous architecture. Among our series of 34 pouch patients, 6 (17.6\%) had advanced adenomas or adenocarcinomas. In the current study, two cases of adenocarcinomas were large, more than $30 \mathrm{~mm}$ in size, and six cases of advanced adenomas were observed among the multiple adenomatous polyps. Groves et al. reported that of 60 patients with adenomas in ileal pouch, 11 (18\%) had advanced histological features [13], and a substantial minority of patients with pouch adenomas developed multiple polyps, large sessile 


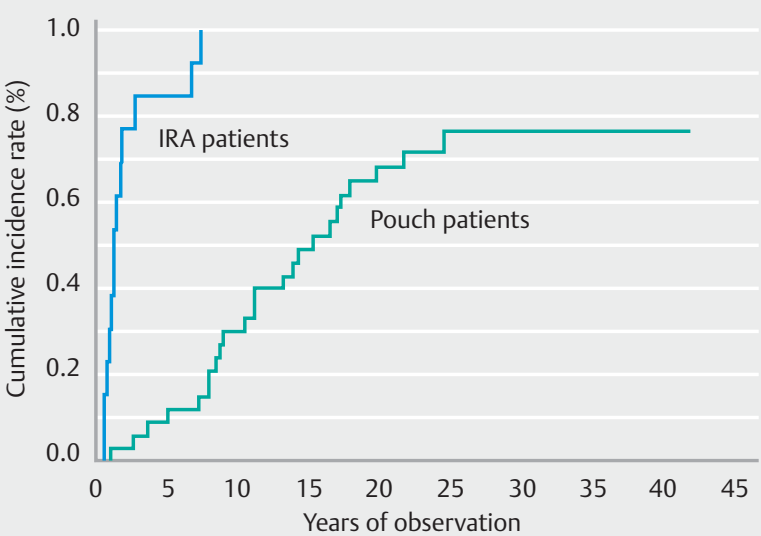

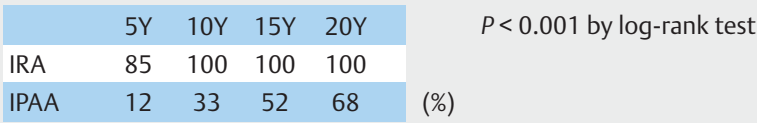

- Fig. 1 Cumulative incidence of adenoma in IRA patients and Pouch patients

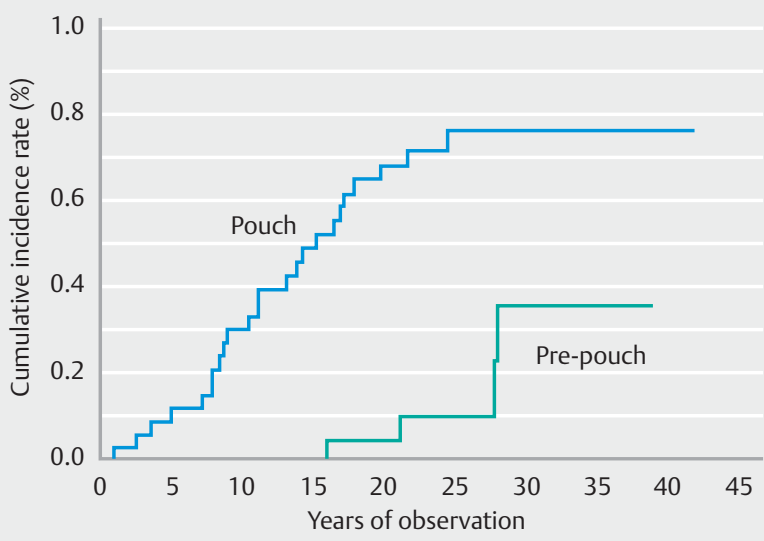

\begin{tabular}{lcccc|} 
& $5 Y$ & $10 Y$ & $20 Y$ & $30 Y$ \\
\hline Pouch & 12 & 33 & 68 & 76 \\
\hline Pre-pouch & 0 & 0 & 4.4 & 36
\end{tabular}

Fig. 2 Cumulative incidence of adenoma in the pre-pouch.

polyps, or adenomas with more advanced histological features. In the current study, there was a significant relationship between maximum size of ileal pouch adenomas and time since surgery $(P=0.0214)$. If pouch adenomas follow the classic adenoma-carcinoma sequence, pouch age becomes one of the risk factors for malignant transformation. Using linear regression analysis in the current study, growth time for the ileal pouch adenoma to reach $10 \mathrm{~mm}$ was 14.1 years. However, in the current series, one case of advanced adenoma developed 8 years after surgery. Tonelli et al. [17] reported that in some patients, development of neoplasia in the ileal pouch may not always follow the classic adenoma-carcinoma sequence, because ileal polyps were not present during endoscopic follow-up until development of pouch carcinoma. Furthermore, the mean inter-

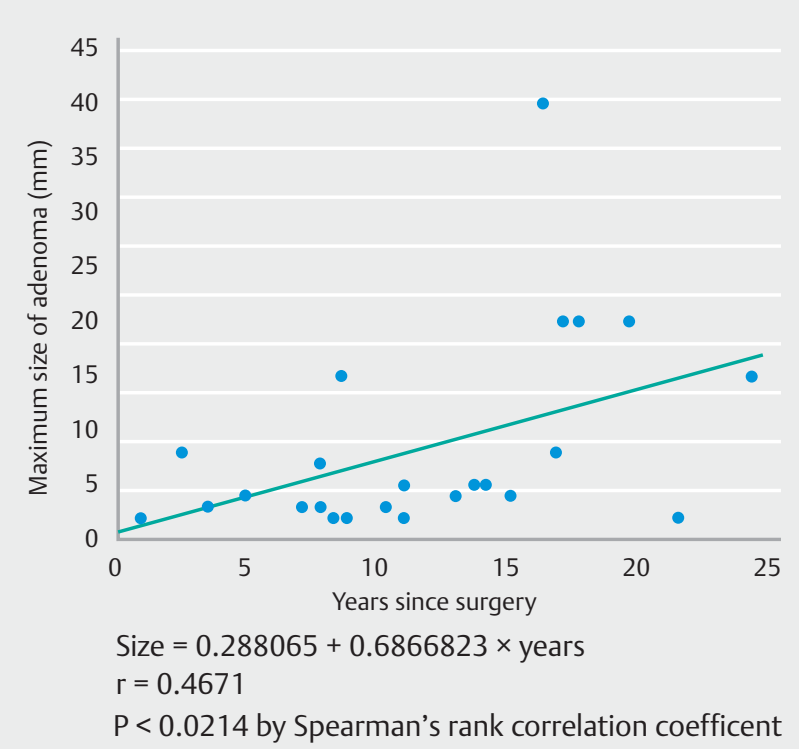

- Fig.3 Relationship between maximum size of ileal pouch adenomas and time since pouch surgery.

val between pouch construction and diagnosis of carcinoma can be very short compared to the colorectum. - Table 3 summarizes the 22 cases of ileal pouch cancer. In some cases, cancer had developed as early as 3 years after pouch construction surgery. It is also remarkable that in several cases, endoscopic surveillance had been done within 1 year before the discovery of cancer. Thus, we must start annual endoscopic surveillance of the ileal pouch at least 3 years after surgery.

In contrast to adenomas in the ileal pouch, development of adenomas in the afferent ileal loop above the pouch (prepouch) may be rare. In this study, there were four ileal adenomas in the pre-pouch in 34 pouch patients (12\%) at median follow-up of 21.6 years after surgery. Cumulative risk of adenoma development in the pre-pouch after surgery was $4.4 \%$ and $36 \%$ at 20 -and 30-year follow-up, respectively, which was significantly lower compared to risk for ileal pouch adenomas $(P<$ $0.001)$. In previous publications, development of pre-pouch adenomas was reported in 10 of 26 (4\%) patients by Wu et al. [11], in two of 20 patients (10\%) by Groves et al. [13], in one of 24 patients (4\%) by Thompson-Fawcett et al. [12], and in eight of 118 patients (6.9\%) by Pommaret et al. [18]. The difference in incidence of adenoma development between the ileal pouch and the pre-pouch may be due to differences in transit time of bowel contents. The mucosa of the ileal pouch may not only be subjected to the tumorigenic consequences of $A P C$ gene mutations [27], but luminal factors due to fecal stasis may also have an important effect. Fecal stasis, such as occurs in a reconstructed pouch, may promote neoplastic changes in the ileal mucosa. Several authors have implicated colonic metaplasia of the ileal mucosa as a precursor for development of ileal adenomas [28-30], and even carcinomas in surgically constructed pouches of patients with FAP [31-33]. Colonic metaplasia was frequently recognized even in earlier descriptions of changes ob- 


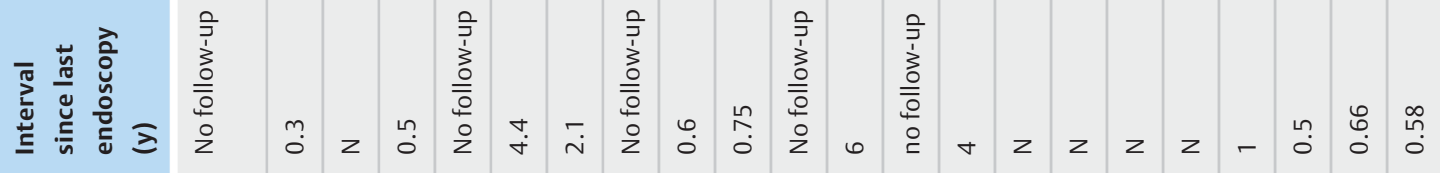

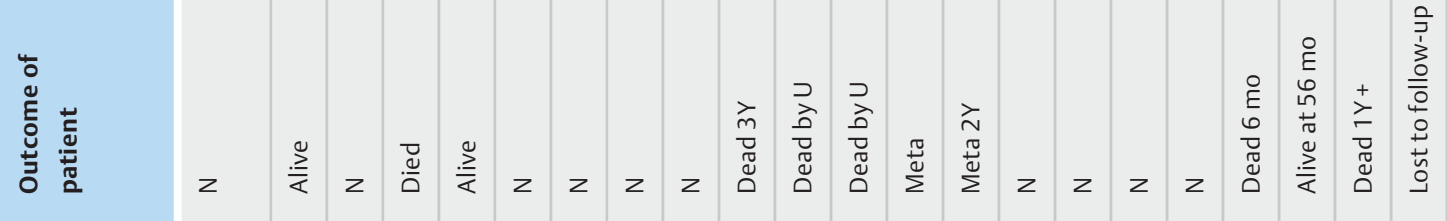

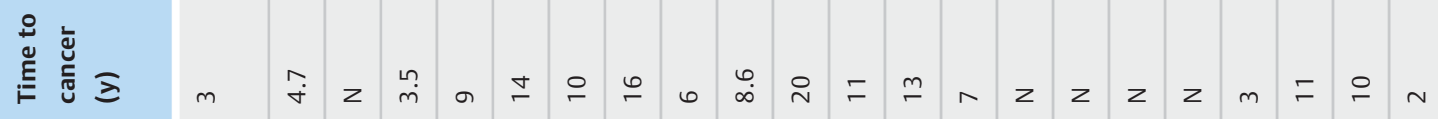

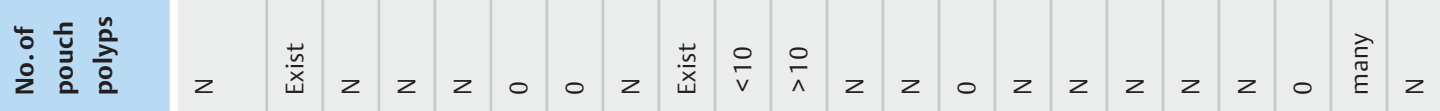

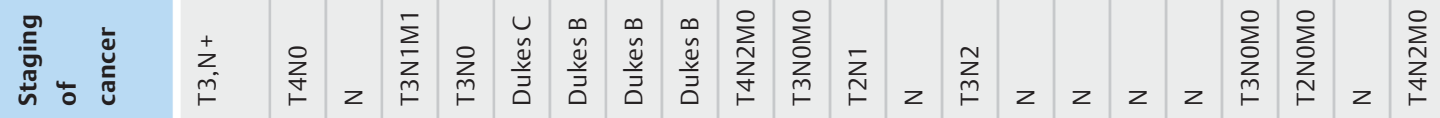

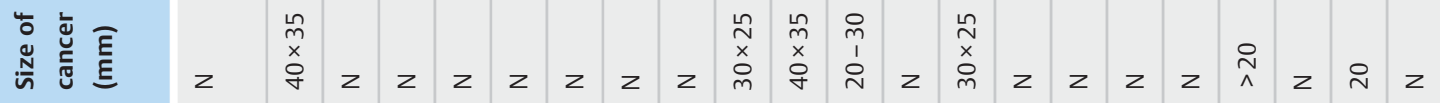

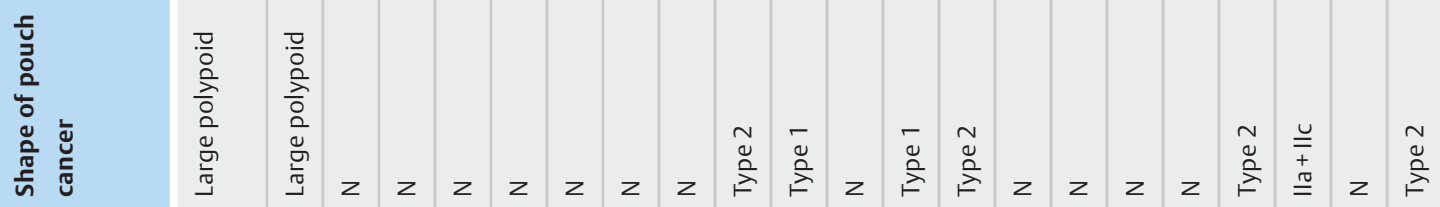

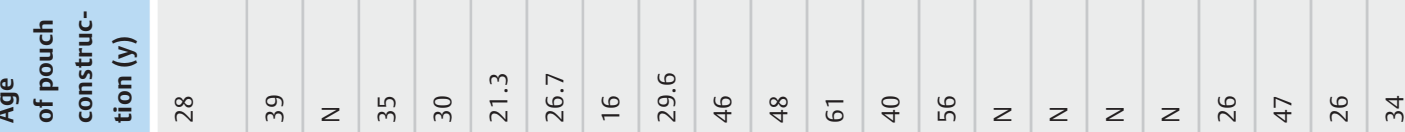

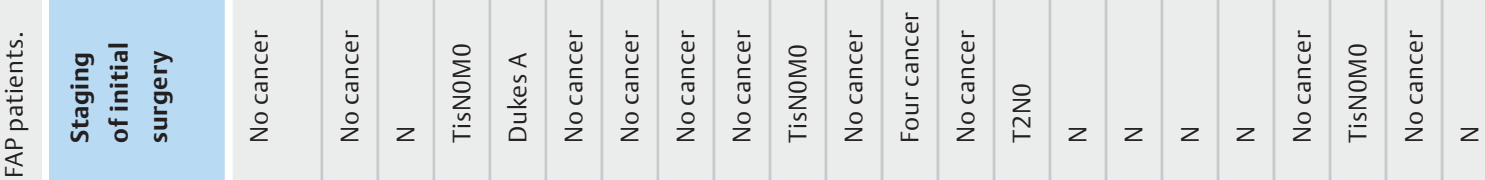

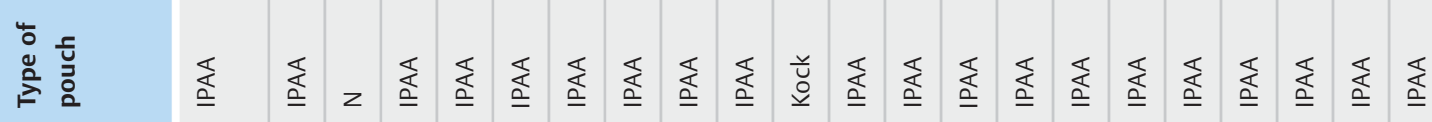

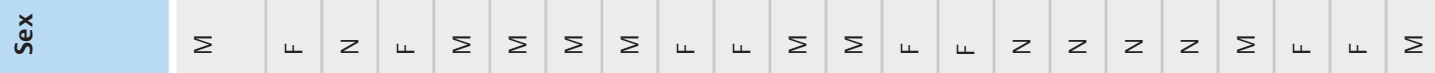


served in ileal pouch mucosa. Some authors have considered colonic metaplasia as an adaptive response of the ileal pouch to its role as a neorectum [34, 35].

At present, it does not seem possible to predict who is at risk for developing polyps in the pre-pouch. Pommaret et al. [18] reported that the only significant risk factor for pre-pouch adenoma was presence of pouch adenomas. The pre-pouch ileum has the same gene as the pouch ileum, and its destiny may be to develop adenomas. However, the cumulative incidence rate was delayed for one or two decades compared to that of the ileal pouch. Furthermore, no case of pre-pouch adenocarcinoma has been reported in the literature. Surveillance of the prepouch ileum may be not as important as that of the ileal pouch.

A drawback of this study is that older patients in the pouch group had not undergone endoscopic surveillance, because the necessity for endoscopic surveillance for patients with IPAA had not been recognized. Another one is that an APC gene mutation study was not performed because it was not permitted by the Japanese health insurance system. Thus, the cumulative incidence rate of adenomas may increase in pouch patients. On the other hand, the strength of this study is that high-definition endoscopes with magnification were used, allowing detection of tiny adenomas in the rectum, ileal pouch, and pre-pouch.

\section{Conclusion}

In conclusion, this study demonstrated a very high cumulative risk of developing adenomas in the ileal pouch, with a lower risk of pre-pouch adenomas. Although pouch adenocarcinomas may be rare, their development may be rapid compared to the colorectum, and thus intensive annual endoscopic surveillance may be appropriate.

\section{Competing interests}

None

\section{References}

[1] Groden J, Thliveris A, Samowitz W et al. Identification and characterization of the familial adenomatous polyposis coli gene. Cell 1991; 66: $589-600$

[2] Bussey HJ, Veale AM, Morson BC. Genetics of gastrointestinal polyposis. Gastroenterology 1978; 74: $1325-1330$

[3] Jagelman DG. Choice of operation in familial adenomatous polyposis. World J Surg 1991; 15: 47-49

[4] Parc YR, Olschwang S, Desaint B et al. Familial adenomatous polyposis: prevalence of adenomas in the ileal pouch after restorative proctocolectomy. Ann Surg 2001; 233: 360 - 364

[5] Kartheuser AH, Parc R, Penna CP et al. Ileal pouch-anal anastomosis as the first choice operation in patients with familial adenomatous polyposis: a ten-year experience. Surgery 1996; 119: 615-623

[6] Nyam DC, Brillant PT, Dozois RR et al. lleal pouch-anal canal anastomosis for familial adenomatous polyposis: early and late results. 1997; 226: 514-519, discussion 519-521
[7] Bess MA, Adson MA, Elveback LR et al. Rectal cancer following colectomy for polyposis. Arch Surg 1980; 115: 460 - 467

[8] De Cosse JJ, Bülow S, Neale K et al. Rectal cancer risk in patients treated for familial adenomatous polyposis. The Leeds Castle Polyposis Group. Br J Surg 1992; 79: 1372-1375

[9] Nugent KP, Phillips RK. Rectal cancer risk in older patients with familial adenomatous polyposis and an ileorectal anastomosis: a cause for concern. Br J Surg 1992; 79: 1204 - 1206

[10] Beart RW Jr., Fleming CR, Banks PM. Tubulovillous adenomas in a continent ileostomy after proctocolectomy for familial polyposis. Dig Dis Sci 1982; 27: $553-556$

[11] Wu JS, McGannon EA, Church JM. Incidence of neoplastic polyps in the ileal pouch of patients with familial adenomatous polyposis after restorative proctocolectomy. Dis Colon Rectum 1998; 41: 552- 556 discussion 556-557

[12] Thompson-Fawcett MW, Marcus VA et al. Adenomatous polyposis develop commonly in the ileal pouch of patients with familial adenomatous polyposis. Dis Colon Rectum 2001; 44: 347 - 353

[13] Groves C], Beveridge IG, Swain DJ et al. Prevalence and morphology of pouch and ileal adenomas in familial adenomatous polyposis. Dis CoIon Rectum 2005; 48: 816 - 823

[14] Moussata D, Nancey S, Lapalus MG et al. Frequency and severity of ileal adenomas in familial adenomatous polyposis after colectomy. Endoscopy 2008; 40: 120 - 125

[15] Friederich P, de Jong AE, Mathus-Vliegen LM et al. Risk of developing adenomas and carcinomas in the ileal pouch in patients with familial adenomatous polyposis. Clin Gastroenterol Hepatol 2008; 6: 1237 1242

[16] Banasiewicz T, Marciniak R, Kaczmarek E et al. The prognosis of clinical course and the analysis of the frequency of the inflammation and dysplasia in the intestinal J-pouch at the patients after restorative proctocolectomy due to FAP. Int J Colorectal Dis 2011; 26: 1197 1203

[17] Tonelli F, Ficari F, Bargellini T et al. Ileal pouch adenomas and carcinomas after restorative proctocolectomy for familial adenomatous polyposis. Dis Colon Rectum 2012; 55: $322-329$

[18] Pommaret E, Vienne A, Lefevre JH et al. Prevalence and risk factors for adenomas in the ileal pouch and the afferent loop after restorative proctocolectomy for patients with familial adenomatous polyposis. Surg Endosc 2013; 27: 3816-22

[19] Tajika M, Niwa Y, Bhatia V et al. Risk of ileal pouch neoplasms in patients with familial adenomatous polyposis. World J Gastroenterol 2013; 19: $6774-84$

[20] Boostrom SY, Mathis KL, Pendlimari R et al. Risk of neoplastic change in ileal pouches in familial adenomatous polyposis. J Gastrointest Surg 2013; 17: $1804-1808$

[21] Goldstein AL, Kariv R, Klausner JM et al. Patterns of adenoma recurrence in familial adenomatous polyposis patients after ileal pouchanal anastomosis. Dig Surg 2015; 32: 421-425

[22] Zahid A, Kumar S, Koorey D et al. Pouch adenomas in familial adenomatous polyposis after restorative proctocolectomy. Int J Surg 2015; 13: $133-136$

[23] Saurin JC, Napoleon B, Gay G et al. Endoscopic management of patients with familial adenomatous polyposis (FAP) following a colectomy. Endoscopy 2005; 37: 499-501

[24] Stoffel EM, Mangu PB, Gruber SB et al. American Society of Clinical Oncology; European Society of Clinical Oncology. Hereditary colorectal cancer syndromes: American Society of Clinical Oncology Clinical Practice Guideline endorsement of the familial risk-colorectal cancer: European Society for Medical Oncology Clinical Practice Guidelines. J Clin Oncol 2015; 33: 209-217 
[25] Kariv R, Rosner G, Fliss-Isakov N et al. Genotype-phenotype associations of APC mutations with pouch adenoma in patients with familial adenomatous polyposis. J Clin Gastroenterol 2019; 53: e54-e60

[26] Church J. Ileoanal pouch neoplasia in familial adenomatous polyposis: an underestimated threat. Dis Colon Rectum 2005; 48: $1708-1713$

[27] Will OC, Robinson J, Günther T et al. APC mutation spectrum in ileoanal pouch polyps resembles that of colorectal polyps. Br J Surg 2008; 95: $765-769$

[28] Shepherd NA, Jass JR, Duval I et al. Restorative proctocolectomy with ileal reservoir: pathological and histochemical study of mucosal biopsy specimens. J Clin Pathol 1987; 40: 601-607

[29] Corfield AP, Warren BF, Bartolo DC et al. Mucin changes in ileoana pouches monitored by metabolic labeling and histochemistry. $\mathrm{Br}$ J Surg 1992; 79: 1209-1212

[30] de Silva H], Millard PR, Kettlewell M et al. Mucosal characteristics of pelvic ileal pouches. Gut 1991; 32: 61-65
[31] Johnson JA, Talton DS, Poole GV. Adenocarcinoma of a Brooke ileostomy for adenomatous polyposis coli. Am J Gastroenterol 1993; 88: $1122-1124$

[32] Johnson $\mathrm{CD}$, White $\mathrm{H}$. Colonic metaplasia with colonic type polyps on an ileostomy stoma in polyposis coli: report a case. Dis Colon Rectum 1988; 31: $405-407$

[33] Nakahara S, Itoha $\mathrm{H}$, lida $\mathrm{M}$ et al. Ileal adenomas in familial polyposis coli: differences before and after colectomy. Dis Colon Rectum 1985; 28: $875-877$

[34] Go PM, Lens J, Bosman FT. Mucosal alterations in the reservoir of patients with Kock's continent ileostomy. Scand J Gastroenterol 1987; 22: $1076-1080$

[35] Lerch MM, Braun ], Harder M et al. Postoperative adaptation of the small intestine after total colectomy and J-pouch-anal anastomosis. Dis Colon Rectum 1989; 32: 600-608 